ORIGINAL ARTICLE

\title{
Reliability and validity of a brief physical activity assessment for use by family doctors
}

\author{
A L Marshall, B J Smith, A E Bauman, S Kaur
}

Br J Sports Med 2005;39:294-297. doi: 10.1136/bjsm.2004.013771

See end of article for authors' affiliations

Correspondence to: Correspondence to:
Dr Marshall, School of Human Movement Studies, University of Queensland, St Lucia, Qld 4072 Australia; alisonm@hms. uq.edu.au

Accepted 20 July 2004
Objective: To evaluate the reliability and validity of a brief physical activity assessment tool suitable for doctors to use to identify inactive patients in the primary care setting.

Methods: Volunteer family doctors $(n=8)$ screened consenting patients ( $n=75$ ) for physical activity participation using a brief physical activity assessment tool. Inter-rater reliability was assessed within one week $(\mathrm{n}=71)$. Validity was assessed against an objective physical activity monitor (computer science and applications accelerometer; $n=42$ ).

Results: The brief physical activity assessment tool produced repeatable estimates of "sufficient total physical activity", correctly classifying over $76 \%$ of cases ( $\kappa 0.53,95 \%$ confidence interval (Cl) 0.33 to 0.72). The validity coefficient was reasonable ( $\kappa 0.40,95 \% \mathrm{Cl} 0.12$ to 0.69 ), with good percentage agreement (71\%).

Conclusions: The brief physical activity assessment tool is a reliable instrument, with validity similar to that of more detailed self report measures of physical activity. It is a tool that can be used efficiently in routine primary healthcare services to identify insufficiently active patients who may need physical activity advice.
$\mathrm{P}$ hysical activity is now recognised as an important health enhancing behaviour. ${ }^{1}$ Primary care doctors have been identified by the community as the preferred source of information about physical activity. ${ }^{2}$ Efficacy studies have repeatedly shown that brief advice from a doctor can result in positive, albeit short term, changes in behaviour. ${ }^{3}$ However, a weakness found in many of the studies reviewed was that interventions were not tested in routine care and in many cases relied on the assistance of third parties (research assistants, receptionists) to identify insufficiently active patients for the doctor to counsel. These methods may be necessary in intervention trials, but for physical activity counselling to be widely adopted in routine practice, family doctors need to be able to measure and monitor their patients' physical activity levels.

In a recent Australian study, ${ }^{4}$ it was difficult for the doctors to have sufficient time to assess their patients' physical activity using a standard physical activity questionnaire. Family doctors are time pressured, thus a brief tool that is reliable and valid is required. The tool also needs to specifically identify whether patients are meeting physical activity guidelines, ${ }^{1}$ which recommend that adults should accumulate at least 30 minutes of moderate intensity physical activity most (preferably all) days of the week, or, for added fitness related benefits, 20 minutes of vigorous intensity physical activity on at least three occasions a week is recommended.

Prochaska et al ${ }^{5}$ developed and evaluated a physical activity assessment tool suitable for use with adolescents in primary care. However, given there are specific physical activity guidelines for adolescents, this tool is not suitable for adults. The aim of this study was to evaluate the reliability and validity of a brief physical activity assessment suitable for family doctors to use to identify insufficiently active adult patients, in need of physical activity advice.

\section{METHODS}

Development of the brief physical activity assessment tool

The validity and reliability of several comprehensive physical activity surveys have been established, ${ }^{67}$ but most are too long and detailed for use by time pressured doctors. Typically these surveys use several questions to assess the frequency and duration of walking and moderate and vigorous intensity physical activity either in the preceding seven days or over a usual week, but the data are then reduced to give an overall estimate of total physical activity, which is often dichotomised to represent either "sufficient" or "insufficient" activity levels based on current guidelines.

The brief physical activity assessment tool under investigation in the study was designed to assess the same dimensions of physical activity as the other surveys, but more efficiently. It consists of two questions, one that assesses the frequency and duration of vigorous intensity physical activity and one that assesses the frequency and duration of moderate intensity physical activities (including walking) undertaken in a "usual" week (Appendix). The "usual" week measurement period was selected instead of the preceding seven days to reduce the possible effect that recent illness may have on reported physical activity.

A scoring algorithm was designed to combine the results of the two questions to identify whether patients are meeting the current physical activity guidelines or not. Thus patients are classified as "sufficiently active" if they report three or more 20 minute sessions of vigorous intensity physical activity or five or more 30 minute sessions of moderate intensity physical activity (including walking) or five or more sessions of any combination of moderate and vigorous intensity physical activity. ${ }^{5}$

\section{Study procedures}

This study was approved by the South Western Sydney Area Heath Service Research Ethics Committee. Eight family doctors volunteered to participate in the study. Patients were recruited to the study over a period of 16 days, by a research assistant. Sequential patients were invited to be involved in the study. Those who were interested gave informed consent and were screened for eligibility by the doctor. Eligible patients were 20-60 years of age, did not suffer from a severe disability or health problem that prevented them from being physically active, and were able to read and understand English. After establishing eligibility, doctors completed the 
brief physical activity assessment. Thereafter, doctors continued with usual patient care. The participating patients were then followed up by the research assistant using the following procedures.

1. To assess inter-rater reliability, all participants whose physical activity level was assessed by the doctor were telephoned up to one week later by the research assistant to re-administer the brief physical activity assessment.

2. To assess criterion validity, participants were asked to wear a physical activity monitor (Computer Science and Applications (CSA) accelerometer) for the following seven days. The CSA monitor has been used repeatedly as a criterion measure of physical activity to validate self report measures of physical activity. ${ }^{67}$

\section{Data analyses}

Inter-rater reliability was determined by comparing the results of the brief physical activity assessment administered by the general practitioner with data collected by the research assistant administering the same instrument.

Criterion validity was assessed by comparing results from the brief physical activity assessment administered by the general practitioner with data collected from the CSA physical activity monitor. CSA data were downloaded into a customised Quick Basic software program, which converts the CSA data (recorded in five minute intervals) into movement counts. The movement counts enable total time spent being active at different intensities to be calculated. Sufficient participation in moderate and vigorous intensity physical activity was defined using the CSA movement count thresholds adopted by Craig et $a l^{6}$ : for moderate intensity activity, the CSA counts had to be $>1952$ and $<5724$ counts/ $\mathrm{min}$; for vigorous intensity activity the CSA counts had to be $>5725$ counts/min. The proportion of participants meeting the sufficient total, moderate and vigorous intensity physical activity criteria based on the CSA data were compared with the findings of the brief physical activity assessment tool.

Participants' data were coded, cross checked, and matched across serial data collection points. Statistical analyses included percentage agreement and $\kappa$ statistics to evaluate criterion validity and inter-rater reliability of the brief physical activity assessment. All data were analysed using SPSS version 9.0 for windows, with a statistical significance level set at 0.05 .

\section{RESULTS}

\section{Participants}

Four hundred patients were screened for eligibility for the study, of whom 220 were excluded because of age $(n=193)$ or disability $(\mathrm{n}=27)$. Seventy five $(\mathrm{n}=56$ female) of the 180 eligible patients agreed to participate in the study. Forty three percent of participants were aged between 41 and 60 years, and most (84\%) had at least a high school education or vocational certificate and were employed (73\%). Almost all were English speaking (93\%) and just under half (49\%) were obese (body mass index $>30 \mathrm{~kg} / \mathrm{m}^{2}$ ). Few participants (3\%) reported that they had received advice from their doctor in relation to physical activity in the past.

Complete self report data were collected from 71 (95\%) participants. Forty two (56\%) of these wore the CSA physical activity monitor for seven days.

\section{Inter-rater reliability}

After the administration of the brief physical activity assessment by the doctor, $25 \%$ of participants were meeting the moderate intensity physical activity guideline, and 32\% of participants were meeting the vigorous intensity physical activity guideline (table 1$)$. Overall, $48 \%(\mathrm{n}=34)$ of participants were classified as "sufficiently" active.

Similar proportions were shown to be meeting the moderate $(28 \%)$ and vigorous (34\%) intensity physical activity guidelines when assessed by the research assistant, and $61 \%$ of patients were classified as sufficiently active. Table 1 shows that the percentage agreement between classifications gained from the two different administrations of the same instrument was high and that $\kappa$ coefficients indicated significant inter-rater agreement $(\mathrm{p}<0.001)$.

\section{Criterion validity}

There was significant agreement between the brief physical activity assessment as administered by the doctor and activity counts recorded by the CSA physical activity monitor in terms of categorising participants as sufficiently active (table 1). Unadjusted percentage agreement data showed reasonable consistency between these measures in terms of classifying whether participants were meeting the vigorous intensity physical activity guideline, but the $\kappa$ statistic for this comparison could not be calculated as no participants recorded adequate vigorous activity counts on the CSA monitors. The $\kappa$ for agreement between the brief assessment tool and the CSA recorded activity in terms of meeting the moderate intensity physical activity guideline was not significant.

\section{DISCUSSION}

Increasingly, family doctors are encouraged to promote physical activity to their patients, thus a valid and reliable tool to enable doctors to be able to identify inactive patients in need of counselling is required. This study evaluated reliability and validity of a brief physical activity assessment that doctors could use during routine consultations to determine if their patients were sufficiently active. The results suggest that this assessment has good inter-rater reliability, with over three quarters of cases being classified as sufficiently active over repeat administrations. Furthermore, there were comparable proportions of participants identified as meeting the vigorous and moderate intensity physical activity guidelines in the two separate assessments.

This brief physical activity assessment has only two questions, making it quick to administer. Being such a concise measure it was not expected to produce comprehensive estimates of physical activity participation, and its reliability and validity coefficients were expected to be weaker than more comprehensive instruments often used in other settings. However, the percentage agreement $(71.4 \%)$ and $\kappa$ statistic $(0.40)$ resulting from the criterion validity assessment for determining sufficient physical activity from this instrument are of a similar magnitude to those observed in other studies that have compared longer self report measures with CSA accelerometers. For instance, percentage agreement for correctly classifying sufficient physical activity between the more comprehensive international physical activity questionnaire and the CSA accelerometers was $73 \%$, with a median correlation coefficient of $0.30{ }^{6}$ This level of agreement is considered acceptable in terms of self reported physical activity assessment. ${ }^{7}$ Similar results were also reported in a study aimed at producing a similar physical activity assessment measure that was suitable for use with adolescents in primary care, ${ }^{5}$ where the percentage agreement between the self report survey and CSA accelerometers was $63 \%$, with a correlation coefficient of 0.40. Although comparisons of measures of agreement between studies with different sample sizes and study populations need to be treated cautiously, they do suggest that the criterion validity of the brief physical activity 
Table 1 Inter-rater reliability and validity of the brief physical activity assessment tool using calculations based on proportions (\% of n) meeting the "sufficient" physical activity criterion and intensity specific guidelines

\begin{tabular}{|c|c|c|c|c|c|}
\hline & $\begin{array}{l}\text { Assessment } \\
\text { administered } \\
\text { by doctor }\end{array}$ & $\begin{array}{l}\text { Assessment } \\
\text { administered by } \\
\text { research assistant }\end{array}$ & $\begin{array}{l}\text { Percentage } \\
\text { agreement }\end{array}$ & $\kappa$ value & $95 \% \mathrm{Cl}$ \\
\hline \multicolumn{6}{|l|}{ Inter-rater reliability $(\mathrm{n}=71)$} \\
\hline Sufficient moderate intensity activity & 25 & 28 & 80 & 0.50 & 0.26 to 0.73 \\
\hline Sufficient vigorous intensity activity & 32 & 34 & 85 & 0.65 & 0.46 to 0.84 \\
\hline \multirow[t]{2}{*}{ Sufficient total activity } & 48 & 61 & 76 & 0.53 & 0.33 to 0.72 \\
\hline & $\begin{array}{l}\text { Assessment } \\
\text { administered } \\
\text { by doctor }\end{array}$ & CSA & $\begin{array}{l}\text { Percentage } \\
\text { agreement }\end{array}$ & к value & $95 \% \mathrm{Cl}$ \\
\hline \multicolumn{6}{|l|}{ Criterion validity ( $n=42$ ) } \\
\hline Sufficient moderate intensity activity & 26 & 26 & 67 & 0.14 & -0.23 to 0.51 \\
\hline Sufficient vigorous intensity activity & 26 & 0 & 74 & - & - \\
\hline Sufficient total activity & 45 & 26 & 71 & 0.40 & 0.12 to 0.69 \\
\hline
\end{tabular}

assessment for classifying people as sufficiently or insufficiently active is as good as longer self report physical activity surveys.

The findings of this study are encouraging, but it is important to consider their limitations. Firstly, the response rate achieved during recruitment indicates that there was a high degree of self selection in the study. This influences the generalisability of the findings to the wider population of general practice patients. Secondly, the sample size was not big enough to examine the reliability and validity of this instrument among distinct subgroups of patients. Thirdly, the instrument has limited sensitivity for detecting the amount of activity performed by patients, as the two questions used ordinal measurement scales. It therefore has limited potential as a means of monitoring modest changes in physical activity participation over time, but could be recommended for initial patient classification.

The development of a brief physical activity assessment suitable for use by family doctors and other healthcare practitioners is a valuable step towards addressing some of the barriers to physical activity counselling in primary care, particularly the perceived lack of resources and skills among practitioners to undertake physical activity interventions. ${ }^{9}$ Given that it is generally accepted now that doctor advice for physical activity is an important component of public health efforts to address this risk factor, this assessment tool may enable more efficient identification of people who may need physical activity advice. The brief physical activity assessment may be incorporated into computer software programs, which are increasingly being used for patient assessment and record keeping, and into activity prescription pads, which have been previously tested and disseminated in Australia. ${ }^{410}$ Indeed, the ongoing presence of physical activity assessments in the healthcare environment may provide a cue for doctors to address this risk factor more regularly as part of health promotion and disease management.

\section{ACKNOWLEDGEMENTS}

The CSA physical activity monitors were borrowed from the US Centers for Disease Control, Atlanta. Dr J Salmon developed the Quick Basic software program used to analyse the CSA data.

\footnotetext{
Authors' affiliations

A L Marshall, School of Human Movement Studies, University of Queensland, St Lucia, Qld, Australia

B J Smith, A E Bauman, S Kaur, University of Sydney, Sydney, Australia

Competing interests: none declared
}

\section{APPENDIX}

(A) How many times a week, do you usually do 20 minutes of vigorous physical activity that makes you sweat or puff and pant? (for example, jogging, heavy lifting, digging, aerobics, or fast bicycling)

- $>3$ times/week

- 1-2 times/week

- none

\section{Score:}

- 4

- 2

- 0

(B) How many times a week, do you usually do 30 minutes of moderate physical activity or walking that increases your heart rate or makes you breath harder than normal? (for example, mowing the lawn, carrying light loads, bicycling at a regular pace, or playing doubles tennis)

- $>5$ times/week

- 3-4 times/week

- 1-2 times/week

- none

Score:

- 4

- 2

- 1

- 0

Total score A + score B:

Score $\geqslant 4=$ "Sufficiently" active (encourage patient to KEEP IT UP)

Score 0-3 = "Insufficiently" active (encourage patient to do MORE)

\section{REFERENCES}

1 United States Department of Health and Human Services. Physical activity and health: a report of the Surgeon General. Atlanta, GA: US Department of and health: a report of the Surgeon
Health and Human Services, 1996.

2 Booth M, Bauman A, Owen N, et al. Physical activity preferences and sources of assistance, and perceived barriers to increase activity among physically inactive Australians. Prev Med 1997;26:131-7.

3 Eakin EG, Glasgow RE, Riley KM. Review of primary care-based physical activity intervention studies. J Fam Pract 2000;49:158-68. 
4 Marshall AL, Booth ML, Bauman AE. Promoting physical activity in Australian general practices: a randomised trial of health promotion advice versus hypertension management. Patient Educ Couns 2005; in press.

5 Prochaska JJ, Sallis JF, Long B, et al. Validity and reliability of physical activity-assessment tools for adolescents in primary care settings. Med Sci Sport Exerc 1999:31:S288.

6 Craig CL, Marshall AL, Sjöström M, et al. International physical activity questionnaire: 12 country reliability and validity. Med Sci Sports Exerc 2003;35:1381-95.

7 Sallis JF, Saelens BE. Assessment of physical activity by self-report: status, limitations, and future directions. Res Q Exerc Sport 2000;71:1-14.

8 US Department of Health and Human Service. Healthy people 2010 (conference edition, in two volumes). Washington, DC: US Dept Health and Human Services, 1996.

9 Smith BJ, Bauman AE, Bull FC, et al. Promoting physical activity through general practice: a controlled trial of written advice and information materials. Br J Sports Med 2000;34:262-7

10 Bull FCL, Schipper ECC, Jamrozik K, et al. Beliefs and behaviour of general practitioners regarding promotion of physical activity. Aust N Z J Public Health 1995; 19:300-4.

\section{............ COMMENTARY}

This is a concise paper reporting the findings of a brief tool for assessing physical activity for use in primary healthcare settings, specifically by general practitioners. It is good research, and, although the sample size is small, appropriate caution is noted. In my view, it is a practical tool which should be used to further the currently limited practice of general practitioners advising on physical activity on a regular basis.

F Bull University of Western Australia, Crawley, WA 6009, Australia; fclbull@cyllene.uwa.edu.au

\section{ELECTRONIC PAGES}

\section{Online case reports}

I he following electronic only articles are published in conjunction with this issue of BJSM (see also pages 253 and 266)

\section{Hepatocellular adenomas associated with anabolic androgenic steroid abuse in bodybuilders: a report of two cases and a review of the literature}

L Socas, M Zumbado, O Pérez-Luzardo, et al

Anabolic androgenic steroids (AAS) are used illicitly at high doses by bodybuilders. The misuse of these drugs is associated with serious adverse effects to the liver, including cellular adenomas and adenocarcinomas. We report two very different cases of adult male bodybuilders who developed hepatocellular adenomas following AAS abuse. The first patient was asymptomatic but had two large liver lesions which were detected by ultrasound studies after a routine medical examination. The second patient was admitted to our hospital with acute renal failure. Ultrasound (US) studies showed mild hepatomegaly with several very close hyperecogenic nodules in liver, concordant with adenomas at first diagnosis. In both cases the patients have evolved favourably and the tumours have shown a tendency to regress after the withdrawal of AAS. The cases presented here are rare but may well be suggestive of the natural course of AAS induced hepatocellular adenomas. In conclusion, sportsmen taking AAS should be considered as a group at risk of developing hepatic sex hormone related tumours. Consequently, they should be carefully and periodically monitored with US studies. In any case, despite the size of the tumours detected in these two cases, the possibility of spontaneous tumour regression must also be taken in account.
(Br J Sports Med 2005;39:e27) http://bjsm.bmjjournals.com/ cgi/content/full/39/5/e27

\section{Posterior sternoclavicular dislocation in a rugby player as a cause of silent vascular compromise: a case report}

A H Mirza, K Alam, A Ali

Background: Approximately 120 cases of posterior sternoclavicular joint (SCJ) dislocation have been documented in the medical literature since it was first described in 1824 by Sir Astley Cooper, a statistic which underlies its relative rarity. It is associated with high energy trauma, and although it may present innocently enough, it is a potentially life threatening injury.

Case and Results: We describe a case in which there was no clinical evidence of complication, although CT imaging revealed complete obstruction of the brachiocephalic vein and impingement of the aorta. This required open reduction and a novel fixation technique was employed. The reduction was stable at 8 month follow up appointment as evidenced by CT scan.

Conclusions: We acknowledge that this type of complication is well recognised but emphasise that it should not be managed complacently. A high index of suspicion is required to determine the presence of serious complications in this type of injury, which may manifest insidiously.

(Br J Sports Med 2005;39:e28) http://bjsm.bmjjournals.com/ cgi/content/full/39/5/e28 